\title{
Mechanical Analysis of Multi-Component Tissues
}

\author{
Frederick H. Silver ${ }^{1}$, Ruchit G. Shah ${ }^{2}$ \\ ${ }^{1}$ Department of Pathology and Laboratory Medicine, Robert Wood Johnson Medical School, Rutgers, The State University of New \\ Jersey, Piscataway, USA \\ ${ }^{2}$ Graduate Program in Biomedical Engineering, Rutgers, The State University of New Jersey, Piscataway, USA \\ Email: fhsilver@hotmail.com
}

How to cite this paper: Silver, F.H. and Shah, R.G. (2017) Mechanical Analysis of Multi-Component Tissues. World Journal of Mechanics, 7, 121-132.

https://doi.org/10.4236/wjm.2017.74011

Received: January 13, 2017

Accepted: April 8, 2017

Published: April 11, 2017

Copyright (C) 2017 by authors and Scientific Research Publishing Inc. This work is licensed under the Creative Commons Attribution International License (CC BY 4.0).

http://creativecommons.org/licenses/by/4.0/

\begin{abstract}
Collagen is the major structural fiber found in mammalian tissues. It is a protein in the form of a triple-helix which is found in several subfamilies, the most abundant of which is the fiber forming group containing Types I, II and III. Type I collagen is found in tendons, skin, cornea, bone, lung and vessel walls. This collagen is thought to give rise to the high tensile strengths of collagen fibers in tissues; in addition, it is actively involved in other physiologic processes such mechanotransduction. However, the non-linear mechanical behavior and viscoelasticity of collagen fibers make analysis of the mechanical properties of tissues complicated. Mechanistically, during mechanical loading, a tensional increase in the $\mathrm{D}$ period is observed with increasing strain that is associated with: 1) molecular elongation at the triple-helical level of structure; 2) increases in the gap distance between the end of one triple-helix and the start of the next one in the microfibril; and 3) molecular slippage. In this paper, we discuss the relationship between collagen hierarchical structure and its non-linear mechanical properties. Using vibrational analysis and optical coherence tomography, it is hoped that the mechanical properties of collagenous tissues can be studied in vivo in order to better understand tissue mechanics and to be better able to offer early diagnosis and differentiation of different disease states.
\end{abstract}

\section{Keywords}

Collagen Fibers, Mechanical Properties, Optical Coherence Tomography, Vibrational Analysis, Tendon, Skin, Cartilage, Elastic Arteries

\section{Introduction}

Collagen fibers form the basic structural components of the extracellular matrix 
(ECM) of vertebrates that serve to: store elastic energy during muscular deformation, transmit stored energy into joint movement, and transfer excess energy from the joint back to the attached muscles for dissipation [1]-[8]. They also act as mechanotransducers by transferring stress borne by the musculoskeleton to the attached cells in order to regulate tissue metabolism, either up- or down, as a result of changes in mechanical loading [2]. Finally, they prevent premature mechanical failure of tissues and limit deformation of most ECMs and organs [2] [3]. Therefore, collagen fiber structure is intimately related to energy storage, transmission, dissipation, and premature mechanical failure of tissues. However, analysis of the basic mechanisms behind this behavior is complicated by the viscoelasticity of collagenous tissues and the presence of other components besides collagen. The viscoelasticity of these tissues leads to non-linear behavior; however, recent studies have identified methods to analyze this behavior [1]-[8].

\section{Collagen Molecular Structure}

Collagen is a protein in the form of a triple-helix which is found in several subfamilies the most abundant of which is fiber forming group containing Types I, II and III. Types I, II, and III form the basic structural units of collagen fibrils and fibers along with some minor types of collagen including types $\mathrm{V}$, IX and XII [9]. Type I collagen is found in tendons, skin, cornea, bone, lung and vessel walls [10]. This collagen is thought to give rise to the high tensile strengths of collagen fibers in tissues; in addition, it actively is involved in other physiologic processes such mechanotransduction [2].

Tendons and skin have been studied extensively in vitro and exhibit non-linear stress-strain behaviors (see Figure 1) [3] [6] [7] [8] [11] [12]. The non-linear behavior is derived from the structural hierarchy of these tissues as well as the domain structure of the triple helix (see Figure 2 and Figure 3 ).

Collagen, when stained with heavy metals, in tissues is recognized by trans-

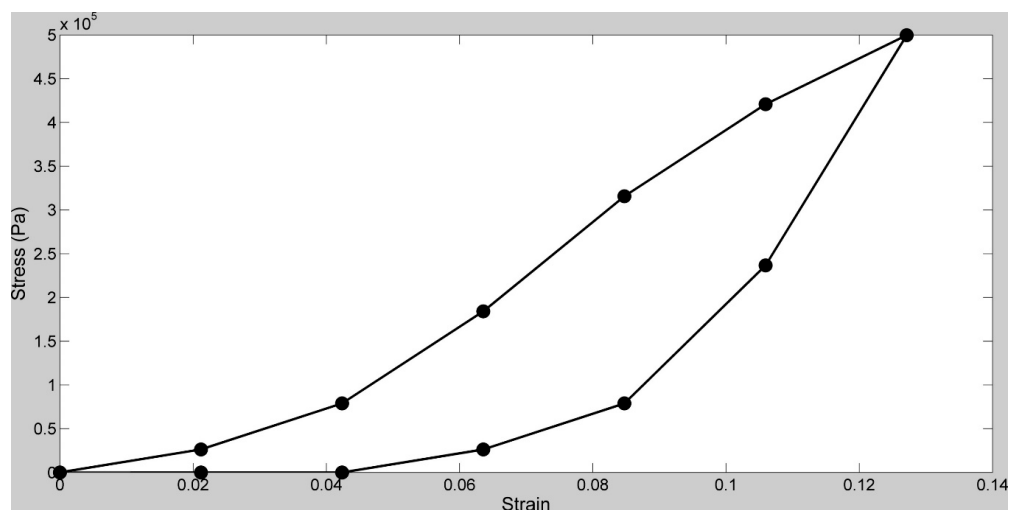

Figure 1. Typical stress-strain curve for decellularized human dermis tested in uniaxial tension. The dermis is loaded in tension in strain increments and the force at each strain is recorded before another strain increment is added. The loading curve is above the unloading curve and the sample returns to zero stress and zero strain after a recovery period of about 30 minutes. Non-linearity is a result of several factors including fiber orientation, loading of other components at low strains (elastic fibers) and viscoelasticity. 


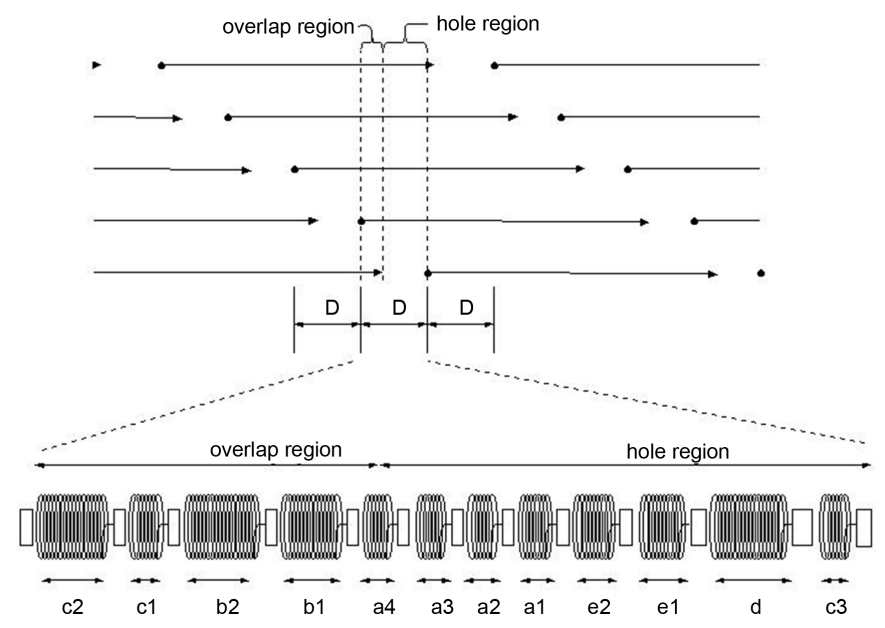

Figure 2. The top portion of this figure illustrates the structure of a five membered microfibrillar unit that is believed to be the repeat unit found in collagen fibrils and fibers that appear as a quasi-hexagonal unit by $\mathrm{x}$-ray diffraction of tendon. In this packing pattern five collagen molecules are staggered by about $22 \%$ of the molecular length of 300 $\mathrm{nm}$ with respect to their nearest neighbors. A space or hole $0.6 \mathrm{D}$ in length ( $\mathrm{D}$ is about 64-67 nm) is left between neighboring molecules. The collagen molecule is $4.4 \mathrm{D}$ long where $\mathrm{D}$ is the stagger between neighboring collagen molecules. The distance $\mathrm{D}$ consists of a overlap zone of $0.4 \mathrm{D}$ and a hole region of $0.6 \mathrm{D}$ as is shown by the vertical dotted lines that are superimposed on the microfibril in the diagram. The overlap and hole regions that make up the $\mathrm{D}$ repeat consist of 13 rigid and 12 flexible domains in the expanded view and are depicted by the rectangles and springs shown, respectively. The 12 flexible regions are identical to the 12 bands denoted c2 through c3 (see Figure 3(d) and Figure 3(e)) that are seen as dark vertical lines across the collagen fibril when collagen is stained with heavy metals and viewed in the electron microscope. The twelve flexible regions are believed to be stretched when collagen fibrils are initially mechanically deformed reflecting experimental increases in the $\mathrm{h}$ spacing that are observed by $\mathrm{x}$-ray diffraction. Further loading causes increases in the D period and molecular and fibrillar slippage. Collagen molecules in tendon are held together in the microfibril with crosslinks that occur at the tail of one molecule (see circle) to the head of a lateral neighboring molecule (see arrowhead). These crosslinks are staggered by a distance of $4 \mathrm{D}$. This Figure was adapted from Silver et al., 2003 [5].

mission microscopy by its regular repeat of the charged amino acid residues. In the quarter-staggered packing pattern, the amino acid sequence of five molecules in cross-section is repeated about every $64 \mathrm{~nm}$, a distance termed the D period (Figure 2 and Figure 3). The D period varies from about 64 to $67 \mathrm{~nm}$ depending on the tissue of origin. In tendon, the D period is about $64 \mathrm{~nm}$ and in skin, it is about $67 \mathrm{~nm}$. At the light microscopic level, collagen fibers in tendon are distinguished from other tissue proteins by the cross striated pattern derived from the crimp (see Figure $3(\mathrm{k})$ ) when viewed under polarized light; however, the fibers in skin, bone and cornea are not crimped but are birefringent (see Figure 3(i), Figure 3(j)).

For instance in tendon, collagen fibril diameters are between 20 and $280 \mathrm{~nm}$ and collagen fibers are between 1 and $300 \mu \mathrm{m}$ wide [4]. Groups of fibril bundles form fascicles that in turn make up the cross-section of a tendon bundle (Figure 4). These structural elements acting in concert giving rise to the mechanical 


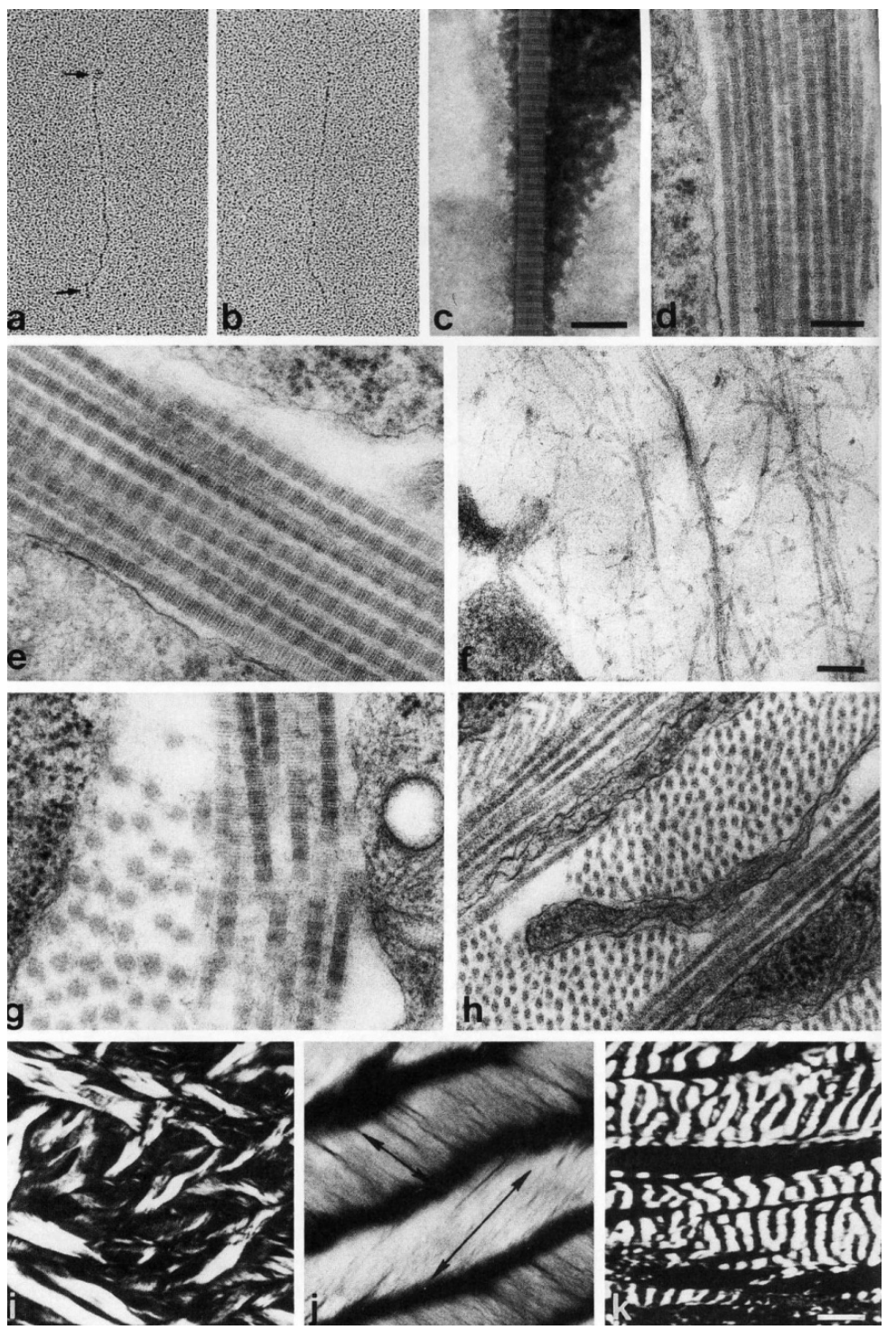

Figure 3. Structural hierarchy of collagen in ECMs. Collagen molecules are semi-flexible rods (bends in (a) (b), that form cross-striated fibrils in tissues with repeat periods between 64 and $67 \mathrm{~nm}$ (c)-(e) or filamentous structures (f). Collagen fibrils are formed in deep recesses of the cell membrane $(\mathrm{g})(\mathrm{h})$ and under polarized light appear as planar biaxial structures in dermis) (i), orthogonal structures in bone (see arrows for collagen molecular directions) in ( $\mathrm{j}$ ) and crimped planar waveforms in tendon $(\mathrm{k})$. This figure was modified from Birk et al., 1991 [20]. The non-linear viscoelastic behavior of collagen fibers is due to geometrical orientation of the collagen fibers and the presence of other components.

properties of tendon. Collagen microfibrils appear to be held together by an interfibrillar matrix containing proteoglycans [13]. Upon deformation and removal of the crimp, the stress-strain curve of tendon slopes upward [14].

\section{Collagen Crosslinking}

In fibril forming collagens, the ability to store, transmit and dissipate energy requires crosslink formation between molecules within a microfibril and between 


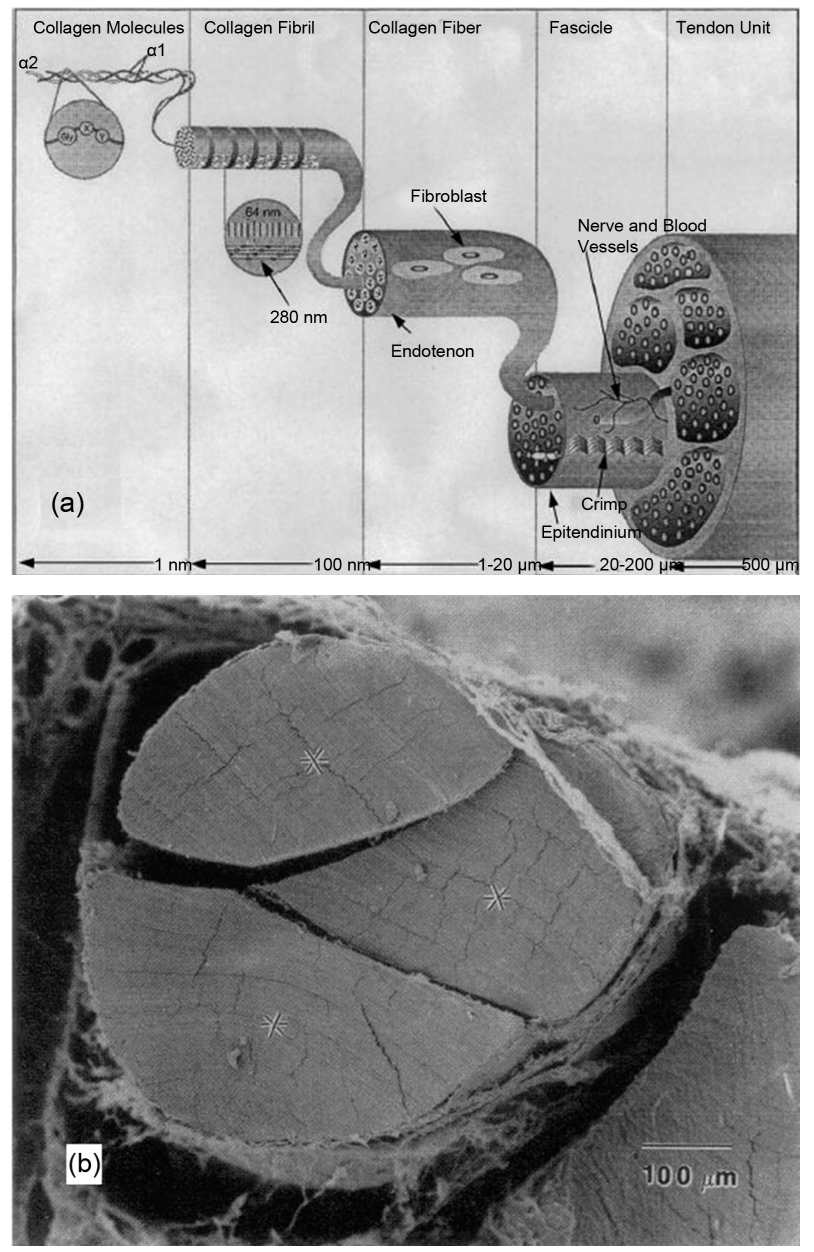

Figure 4. Structural hierarchy in rat tail tendon. (a) This diagram illustrates the relationship between collagen molecules, fibrils, fibers, fascicles, and tendon units. Although the diagram does not show fibril subunits, collagen fibrils appear to be self-assembled from intermediates integrated within the fibril termed microfibrils; (b) Scanning electron micrograph of a rat-tail tendon fiber showing the fascicles (see asterisks) that make up the tendon unit. In human tendons there are many collagen fascicles that make up a tendon bundle. Figure adapted from Silver et al., 2003 [5].

microfibrils and other structural units. These crosslinks include lysine and hydroxylysine derivatives and other amino acid residues including histidine that are at the ends of the molecules (see Figure 2) [4]. Additional crosslinking also occurs during aging and involves glucose molecules [9]. The stiffening and poor energy dissipation of collagen fibers associated with aging of the skin involves collagen fiber fragmentation by exposure to UV light and glucose derived crosslinking. This leads to loss of the ability of ECMs to transmit and dissipate energy and is associated with loss of auxiliary pumping efficiency of the elastic arteries during pulsatile blood flow in the cardiovascular system [15].

\section{Viscoelastic Behavior of Collagen Fibers}

Collagen fibers are viscoelastic and exhibit non-linear mechanical behavior in tissues such as human dermis (see Figure 1). Since the stress-strain curve is non- 
linear, the modulus is traditionally calculated from the tangent to the stressstrain curve at a particular strain. Since stress-strain curves for tissues typically curve upward, i.e., strain harden, this makes analysis of the modulus, strain and strain-rate dependent. Viscoelasticity may be important in resisting impact loads especially in the musculoskeleton; however, it complicates the understanding of ECM behavior since most real-time measurements made on these tissues contain both elastic and viscous contributions [3]. The elastic behavior varies from as high as about $75 \%$ of the total stress for tendon to as low as about $50 \%$ for skin depending on the collagen fiber orientation, rate of loading and the quantity of other tissue constituents [3].

A number of excellent studies have been published that have helped in the interpretation of the stress-strain behavior of tendon at the molecular and fibrillar levels. Much of our current understanding of the relationship between hierarchical structure and viscoelastic behavior of ECMs is based on studies of the mechanical properties of developing and mature tendons [16] [17] [18] [19]. The properties of developing tendon rapidly change just prior to the onset of locomotion. The maximum total stress that can be borne by a 14 day old embryonic chick leg extensor tendon is about $2 \mathrm{MPa}$ and increases to $60 \mathrm{MPa}$ two days after birth [18] [19]. This rapid increase in tensile stress by tendon occurs without large changes in its hierarchical structure [18] [19]. In this case, the collagen fibril length appears to be more important for energy storage and for increased ultimate tensile strength than fibril diameter; but the two parameters are linked together since fibrils have been shown to grow in length by lateral fusion of fibril bundles [5] [16] [19] [20] (see Figure 3(h)).

Mechanistically, during mechanical loading, a tensional increase in the D period is observed with increasing strain that is associated with: 1) molecular elongation at the triple-helical level of structure in the flexible spring-like regions (see Figure 2); 2) increases in the gap distance between the end of one triple-helix and the start of the next one in the microfibril; and 3) molecular slippage [21]. Molecular stretching occurs at lower stresses followed by increases in the gap spacing and molecular sliding that occur at higher stresses [22]. It is believed that molecular stretching occurs first in the regions devoid of proline and hydroxyproline as diagrammatically represented by the springs in Figure 2. These springs represent the charged regions of the sub-bands in the D period that are shown in transmission electron micrographs of collagen fibrils in tissues (compare Figure 2, Figure 3(d), Figure 3(e) and Figure 3(g)).

The time-dependent behavior of tendon makes it difficult to interpret stressstrain relationships for these tissues. However using incremental stress-strain curves, the elastic and viscous behaviors can be separated and analyzed in terms of tissue structure [3]. The viscoelastic properties of ECMs have been obtained by constructing incremental stress-strain curves for a variety of tissues including tendon [2] [3] (see Figure 5). Such incremental stress-strain curves are derived for tendon and other ECMs by stretching the tissue in a series of strain increments and then allowing the stress to relax to an equilibrium value at each strain 


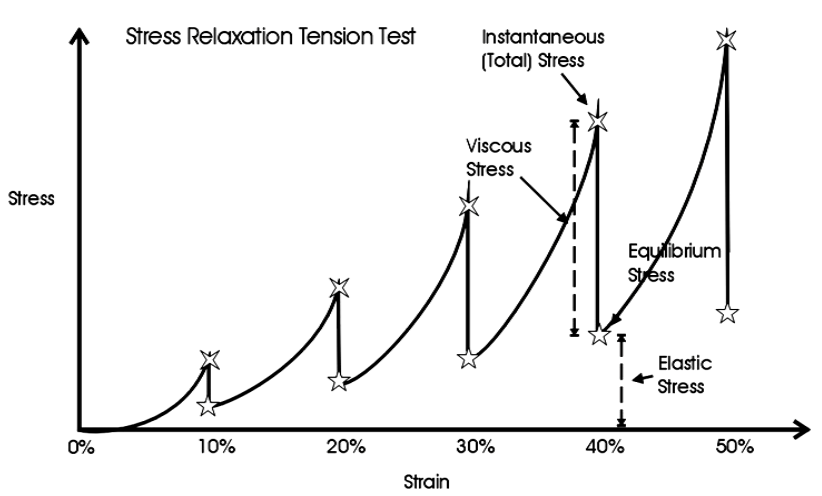

(a)

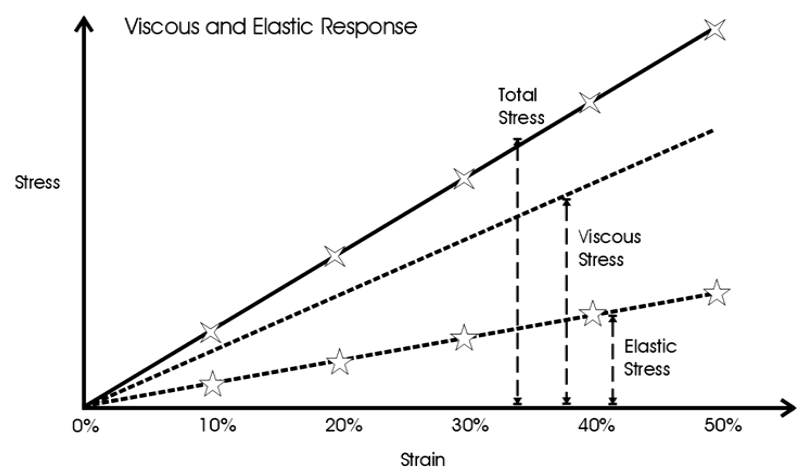

(b)

Figure 5. Incremental stress-strain curves for ECMs tested in tension. (a) A strain increment is applied to the ECM and the initial stress is measured. The strain increment varies from about $2 \%$ for tendon to about $10 \%$ for skin. The stress is allowed to relax at room temperature until an equilibrium value is reached. The process is repeated until the sample fails; (b) Plots of all the initial (total) and equilibrium stresses are made versus strain as well as plot of the total stress minus the equilibrium stress versus strain. The equilibrium stress versus strain curve is equivalent to the elastic stress-strain curve while the difference between the total and equilibrium stress is the viscous stress. This figure was adapted from Silver, 2006 [2].

increment before another strain increment is added (Figure 5(a)) [3]. By subtracting the elastic stress (equilibrium stress value) from the initial or total stress value, the viscous stress is obtained. By plotting the equilibrium stress versus strain and the total stress minus the equilibrium stress versus strain (Figure 5(b)) we get elastic and viscous stress-strain curves for tendon [2] (Figure 6). From these curves and the literature, important information can be obtained concerning the mechanism of stretching and sliding of the collagen molecules and fibrils that make up the structure of tendon fibers [2]. Note in the absence of the crimp the relationship between elastic stress and strain is approximately linear (see Figure 6). It turns out that the slope of the elastic stress-strain curve is proportional to the elastic modulus of the collagen molecule [2] [5], while the viscous stress at a particular strain is a measure of the fibril length [2] [5]. An estimate of the elastic modulus of the collagen molecule is obtained by dividing the slope of the elastic stress-strain curve by the collagen content and by the ratio of the molecular strain (change in $\mathrm{h}$ spacing-axial rise per amino acid residue along 


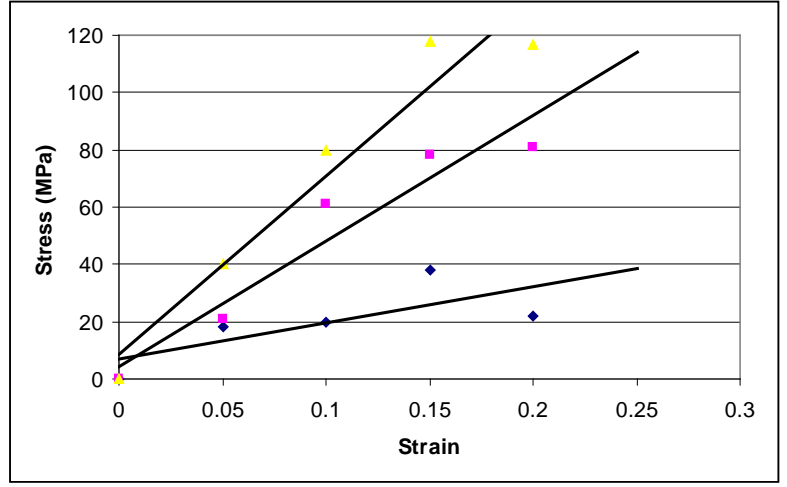

Figure 6. Total, elastic and viscous stress-strain curves for rat-tail tendon fibers obtained from incremental stress-strain testing in uniaxial tension. This figure shows that the elastic stress-strain curve (squares) is approximately linear with strain and is above the viscous curve (triangles). This diagram illustrates that more energy is stored during tensile deformation of tendon than is dissipated during stretching. Prior to crosslinking the viscous stress-strain curve is above the elastic curve indicating that energy storage in collagenous tissues requires adequate crosslinking between the collagen molecules in the quarter staggered packing pattern shown in Figure 2. This Figure was adapted from Silver et al., 2003 [5].

the molecule backbone) divided by the macroscopic strain ( 0.1 for tendon) [2]. Using this approach a value of between 7 and $8 \mathrm{GPa}$ for the elastic modulus of the collagen molecule is found for rat tail tendon collagen fibers [2]. Elastic moduli (Table 1) for tendon, skin and cartilage have been shown to be strain rate independent in tension [2]. Collagen fibril lengths calculated from the viscous stress and hydrodynamic theory [2] range from about $20 \mu \mathrm{m}$ for developing tendon to in excess of $1 \mathrm{~mm}$ for adult tendons (see Table 1) [2].

\section{Non-Destructive Methods for Studying Mechanical Behavior of Collagen Fibers and Tissues}

The ability to monitor the mechanical properties of collagen fibers and ECMs in vivo is an important measurement needed for early diagnosis of disease and the ability to follow disease progression. Physicians daily "palpate" changes in the properties of tissues associated with tumors and calcification suggesting that there are major changes in the structure and properties of collagen and ECMs during disease processes. It is essential that clinicians be able to assess the changes at the collagen fibril and fiber levels of structure to accurately diagnose and treat diseases such as cancer. Several new mechanical methods have been developed to try to discern these changes early in the disease process. It is essential that these methods be validated so that the properties measured have some meaning.

There are several new methods that have been evaluated in the literature to study the mechanical properties of tissues in vivo such as magnetic resonance elastography (MRE), ultrasound elastography (UE), optical coherence tomography (OCT), ocular response analysis (ORA), optical coherence elastography (OCE), and OCT with vibrational analysis [23]. Classical methods such as con- 
Table 1. Estimated elastic moduli for collagen based on elastic stress measurements for various ECMs [2].

\begin{tabular}{cll}
\hline Molecule & \multicolumn{1}{c}{ Tissue } & \multicolumn{1}{c}{ Elastic Modulus (GPa) } \\
\hline \multirow{2}{*}{ Type I } & Self-assembled & 6.51 \\
Rat tail tendon & 7.69 \\
Turkey tendon & 4.2 (no mineral) \\
Types I and III & Skin & 4.22 (mineral 0.245) \\
& & 7 (surface parallel) \\
Type II & Articular cartilage & 2.21 (surface perpendicular) \\
& 4.91 (whole parallel) \\
& & 1.52 (whole perpendicular) \\
& Skin & 0.092 (whole perpendicular) \\
Elastic Fibers & Vessel wall & 0.01 \\
\hline
\end{tabular}

stant rate-of-strain deformation as well as incremental stress-strain analysis are useful but prove to be too destructive to tissue and therefore have limited value for measuring tissue properties in vivo. Vibrational analysis in combination with optical coherence tomography has been used to measure the mechanical properties of collagenous tissues. Results compare favorably with the results of incremental stress-strain curves measured in tension in the classical manner [6] [7].

\section{Vibrational Analysis and Ocular Coherence Tomography (OCT)}

We have used vibrational analysis in combination with optical coherence tomography to study the mechanical properties of decellularized human dermis and Silicone rubber models [6] [7] (see Figure 7). Using this technique the vibrational modulus of a material and the tensile modulus can be measured at the same time. In the experiment, an acoustic vibration is applied to the sample under tension and the resonant frequency is obtained by measuring the displacement as a function of frequency. The resonant frequency is found by determining the frequency at which the maximum displacement occurs. The resonant frequency squared is related to the modulus as described previously [6] [7]. This method has been shown to give moduli values that correlate with tensile moduli as shown in Figure 8 [7]. Vibrational analysis of tissues is particularly useful since the modulus can be calculated at a single strain as opposed to being determined from the tangent to the stress-strain curve, which requires measurements at several points.

\section{Conclusions}

Collagen fibers are the structural elements found in vertebrate tissues that 


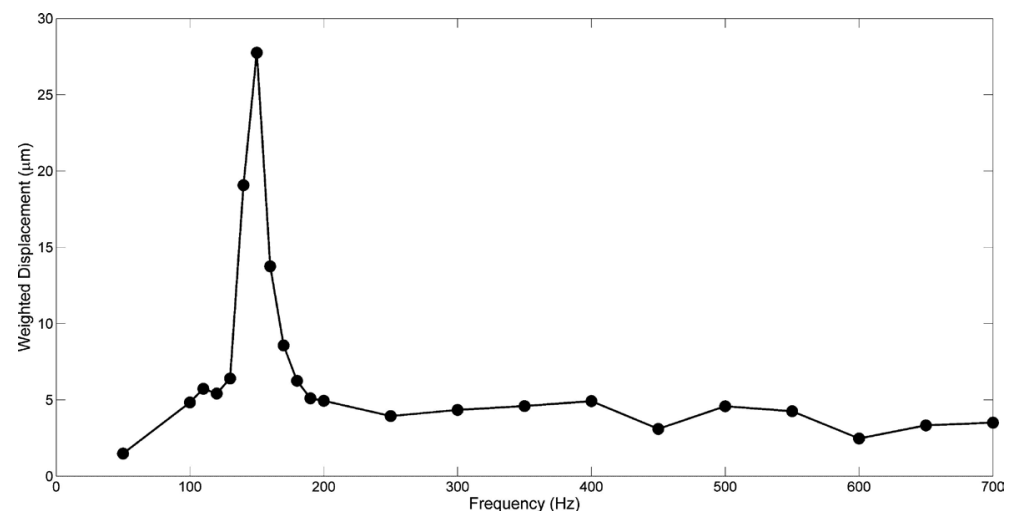

Figure 7. Vibrational analysis of decellularized dermis. Determination of the resonant frequency from measurement of the maximum displacement of a sample vibrated between 0 and $700 \mathrm{~Hz}$. Note the maximum displacement is measured and converted into a vibrational modulus using the calibration curve shown in Figure 8. The modulus calculated from vibrational studies was calculated from the resonant frequency as described by Shah et al., 2016 and 2017 [7] [8].

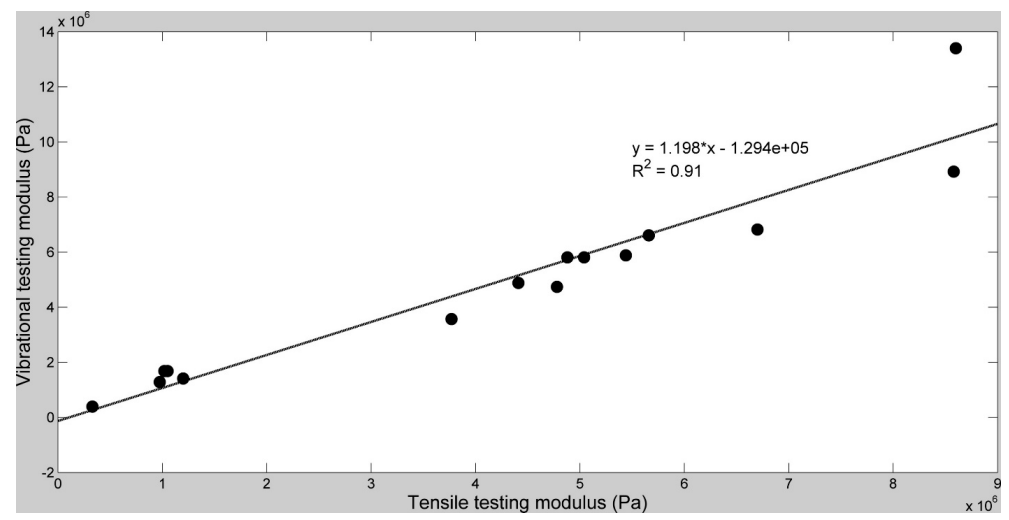

Figure 8. Calibration curve of the modulus determined from vibrational studies (obtained from measuring the frequency at which the maximum displacement occurs) versus the tensile modulus determined from force versus extension curves for decellularized dermis and silicone rubber. The tensile modulus was measured from the slope of incremental stress-strain curves and the modulus from vibrational studies was obtained from the resonant frequency as described by Shah et al., 2016 and 2017 [7] [8].

transmit forces, store and dissipate energy. Collagen fibers limit the deformation of tendon and other load bearing tissues and have a hierarchical structure that includes collagen molecules, microfibrils, fibrils, fibers and fascicles. Collagen molecules are packed into a quarter-stagger packing pattern with neighboring molecules staggered by multiples of $\mathrm{D}$, which is about $22 \%$ of the molecular length. During mechanical deformation collagen molecules are stretched as well as the gap region of the $\mathrm{D}$ period. The elastic modulus of collagen molecules can be calculated from the slope of the tensile stress-strain curve after correction for the collagen content and change in axial rise per amino acid residue that occurs during tensile deformation. In tissues such as tendon, the low modulus region occurs due to unfolding of the crimped collagen fibers. The elastic modulus values calculated from measurements made on several ECMs range from about 2 to 
$8 \mathrm{GPa}$. The differences in the values measured reflect differences in collagen concentration, orientation and the presence of other materials. The low modulus region involves geometric alignment of collagen fibers with the tensile axis as well as stretching of other components in series with the collagen fibers. At larger strains, molecules and fibrils slide by each other which lead to energy losses and viscous effects.

Using vibrational analysis in concert with OCT the modulus of ECMs containing collagen fibers can be measured by determination of the resonant frequency. Measured resonant frequencies for decellularized dermis and Silicone rubber when converted into moduli correlate well with the tensile moduli measured for the same samples. Ultimately, it is hoped to measure the vibrational moduli of tissues and implants in vivo to better understand collagen fiber behavior in health and disease.

\section{References}

[1] Silver, F.H. and Landis, W.J. (2008) Viscoelasticity, Energy Storage and Transmission and Dissipation by Extracellular Matrices in Vertebrates. In: Fratztl, P., Ed, Collagen, Structure and Mechanics, Springer, Chapter 6, New York, 133-154. https://doi.org/10.1007/978-0-387-73906-9_6

[2] Silver, F.H. (2006) Mechanosensing and Mechanochemical Transduction in Extracellular Matrix, Biological, Chemical, Engineering and Physiological Aspects. Springer, New York.

[3] Dunn, M.G. and Silver, F.H. (1983) Viscoelastic Behavior of Human Connective Tissue: Relative Contribution of Viscous and Elastic Components. Connective Tissue Research, 12, 59-70. https://doi.org/10.3109/03008208309005612

[4] Silver, F.H., Kato, Y.P., Ohno, M. and Wasserman, A.J. (1992) Analysis of Mammalian Connective Tissue: Relationship between Hierarchical Structures and Mechanical Properties. Journal of Long-Term Effects of Medical Implants, 2, 165-172.

[5] Silver, F.H., Freeman, J.W. and Seehra, G.P. (2003) Collagen Self-Assembly and Development of Matrix Mechanical Properties. Journal of Biomechanics, 36, 15291553. https://doi.org/10.1016/S0021-9290(03)00135-0

[6] Shah, R., Pierce, M.C. and Silver, F.H. (2017) A Method for Non-Destructive Mechanical Testing of Tissues and Implants. Journal of Biomedical Materials Research Part A, 105, 5-22.

[7] Shah, R., Pierce, M.C. and Silver, F.H. (2016) Morphomechanics of Dermis-A Method for Non-Destructive Testing of Collagenous Tissues. Skin Research and Technology.

[8] Shah, R., DeVore, D., Pierce, M.C. and Silver F.H. (2017) Vibrational Analysis of Implants and Tissues: Calibration and Mechanical Spectroscopy of Multi-Component Materials. Journal of Biomedical Materials Research Part A.

[9] Ricard-Blum, S. (2011) The Collagen Family. In: Hynes, R. and Yamada, K., Eds., Cold Spring Harbor Perspectives in Biology, Cold Spring Harbor Press, New York, $1-19$.

[10] Hulmes, D.J.S. (2008) Collagen Diversity, Synthesis and Assembly. In: Fratzl, P., Ed, Collagen, Structure and Mechanics, Springer, New York, Chapter 2, 15-47. https://doi.org/10.1007/978-0-387-73906-9_2

[11] Yamada, H. (1970) Strength of Biological Materials. Williams and Wilkins, Baltimore, MD. 
[12] Fung, Y.C. (1973) Biomechanics: Mechanical Properties of Living Tissue. 2nd Edition, Springer, New York.

[13] Scott, J.E. (1992) Supramolecular Organization of Extracellular Matrix Glycosaminoglycans in Vitro and in Tissues. The FASEB Journal, 6, 2639-2645.

[14] Diamant J., Keller, A., Baer, E., Litt, M. and Arridge, R.G. (1972) Collagen: Ultrastructure and Its Relation to Mechanical Properties as a Function of Ageing. Proceedings of the Royal Society of London B, 180, 293-315.

https://doi.org/10.1098/rspb.1972.0019

[15] Horvath, I., Foran, D.J. and Silver, F.H. (2005) Energy Analysis of Flow Induced Harmonic Motion in Blood Vessel Walls. Cardiovascular Engineering, 5, 21-28. https://doi.org/10.1007/s10558-005-3070-Z

[16] Torp, S., Baer, E. and Friedman, B. (1974) Effects of Age and of Mechanical Deformation on the Ultrastructure of Tendon. Proceedings of the Colston Conference, University of Bristol, Bristol, 26, 223-250.

[17] McBride, D.J. (1984) Hind Limb Extensor Tendon Development in the Chick: A Light and Transmission Electron Microscopic Study. M.S. Thesis, Rutgers University, NJ.

[18] McBride, D.J., Hahn, R. and Silver, F.H. (1985) Morphological Characterization of Tendon Development during Chick Embryogenesis: Measurement of Birefringence Retardation. International Journal of Biological Macromolecules, 7, 71-76. https://doi.org/10.1016/0141-8130(85)90034-0

[19] McBride, D.J., Trelstad, R.L. and Silver, F.H. (1988) Structural and Mechanical Assessment of Developing Chick Tendon. International Journal of Biological Macromolecules, 10, 194-200. https://doi.org/10.1016/0141-8130(88)90048-7

[20] Birk, D.E., Silver, F.H. and Trelstad, R.L. (1991) Matrix Polymerization. In: Hay, E.D., Ed., the Cell Biology of the Extracellular Matrix, 2nd Edition, Academic Press, New York, 221-254. https://doi.org/10.1007/978-1-4615-3770-0_8

[21] Sasaki, N., Shukunami, N., Matsushima, N. and Izumi, Y. (1999) Time-Resolved X-Ray Diffraction from Tendon Collagen during Creep Using Synchrotron Radiation. Journal of Biomechanics, 32, 285-292. https://doi.org/10.1016/S0021-9290(98)00174-2

[22] Folkhard, W., Geercken, W., Knorzer, E., Mosler, E., Nemetschekgansler, H., Nemetschek, T. and Koch, M.H.J. (1987) Structural Dynamic of Native Tendon Collagen. Journal of Molecular Biology, 193, 405-407. https://doi.org/10.1016/0022-2836(87)90228-2

[23] Silver, F.H. and Shah, R. (2016) Measurement of Mechanical Properties of Natural and Engineered Implants. Advances in Tissue Engineering and Regenerative Medicine, 1, 1-9. 
Submit or recommend next manuscript to SCIRP and we will provide best service for you:

Accepting pre-submission inquiries through Email, Facebook, LinkedIn, Twitter, etc. A wide selection of journals (inclusive of 9 subjects, more than 200 journals)

Providing 24-hour high-quality service

User-friendly online submission system

Fair and swift peer-review system

Efficient typesetting and proofreading procedure

Display of the result of downloads and visits, as well as the number of cited articles Maximum dissemination of your research work

Submit your manuscript at: http://papersubmission.scirp.org/

Or contact wjm@scirp.org 